\title{
Fauna e sua relação com atributos edáficos em Lages, Santa Catarina - Brasil
}

\author{
Fauna and its relation to edaphic attributes in Lages, Santa Catarina - Brazil
}

\author{
Pâmela Niederauer Pompeo ${ }^{1}$, Marcielli Aparecida Borges dos Santos ${ }^{2}$, Josieli Pietro Biasi², Silvane de Fatima Siqueira ${ }^{1}$, \\ Marcio Gonçalves da Rosa ${ }^{3}$, Carolina Riviera Duarte Maluche Baretta ${ }^{4}$, Dilmar Baretta ${ }^{5 *}$

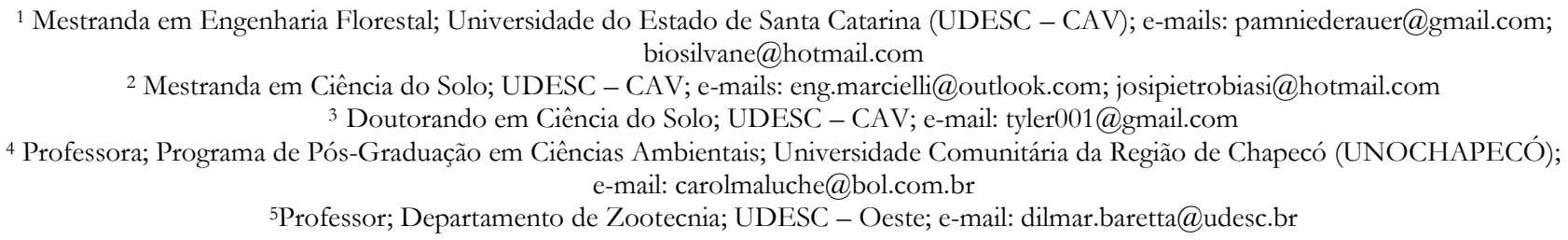

Artigo enviado em 29/04/2016, aceito em 17/06/2016 e publicado em 13/10/2016.

RESUMO - A fauna edáfica é sensível às perturbações ambientais e sua resposta pode indicar o estado de conservação do solo em locais com diferentes usos e manejos. O objetivo deste estudo foi avaliar a diversidade de grupos da fauna invertebrada e sua relação com atributos edáficos em três usos da terra: Floresta Nativa (FN), Reflorestamento de Pinus (RP) e Campo Nativo Melhorado (CNM), no município de Lages, SC. A amostragem consistiu em três pontos distanciados entre si por 30 metros, estabelecidos ao longo de transectos, em cada sistema. Avaliaram-se os atributos físicos e químicos do solo e da abundância e diversidade da fauna, coletada pelos métodos Pitfall traps e Tropical Soil Biology and Fertility. Os dados foram submetidos à análise estatística multivariada. Identificaram-se 1.210 organismos do solo, pertencentes a 17 grupos taxonômicos, sendo, os mais representativos Collembola e Coleoptera, independente do uso. A FN apresentou maior riqueza e diversidade da fauna edáfica em comparação aos outros sistemas. Os maiores teores de matéria orgânica e pH demonstraram relação com Oligochaeta, Enchytraeidae e Collembola. A umidade do solo contribuiu para explicar a abundância dos grupos em CNM e FN. Contudo, a fauna mostrou respostas diferentes na sua distribuição para cada uso da terra, logo, as variáveis ambientais podem limitar o estabelecimento dos invertebrados mais frequentes no solo. Os sistemas FN e CNM apresentaram melhores condições dos atributos edáficos e por isso maior biodiversidade, quando comparados ao RP.

Palavras-chave - biodiversidade, conservação do solo, sistemas de manejo.

\begin{abstract}
The soil fauna is sensitive to environmental disturbances, their response may indicate the state of soil conservation in different uses and management practices. The aim of this study was to evaluate the diversity of invertebrate fauna groups and its relationship with edaphic attributes in three land uses: Native Forest (NF), Pinus Reforestation (PR) and Native Improved Grassland (NIM), in Lages, SC - Brazil. Sampling consisted of three points distanced each other 30 meters, established along transects, on each system. The evaluations were physical and chemical soil attributes and the abundance and diversity of fauna, collected by methods Pitfall traps and Tropical Soil Biology and Fertility. The data were subjected to multivariate statistical analysis. They identified 1.210 soil organisms belonging to 17 taxonomic groups. The most representative groups were Collembola and Coleoptera, independent of the use. The NF has introduced greater richness and diversity of soil fauna in comparison to other LUS. The highest levels of organic matter and soil $\mathrm{pH}$ showed a relationship with Oligochaeta, Enchytraeidae and Collembola. The values of moisture contributed to explain the greater abundance of groups in NIM and NF. However, the soil fauna showed different responses in its distribution to each land use, being that the environmental variables may limit the establishment of most frequent belowground invertebrates. NF systems and NIM presented better terms of edaphic attributes and therefore greater biodiversity, when compared to the PR.
\end{abstract}

Key-words - biodiversity, soil conservation, management systems. 


\section{INTRODUÇÃO}

O solo em condições naturais tende a apresentar alta diversidade de organismos e por essa razão é considerado um sistema complexo e dinâmico, com mecanismos ainda pouco compreendidos. Possui, em sua composição, agentes biológicos como as raízes das plantas, microbiota e a fauna do solo, sendo esta última responsável por diversas atividades inerentes a qualidade dos solos (BARDGETT; PUTTEN, 2014).

A fauna do solo é composta por organismos que passam toda ou parte de sua vida nesse ambiente e para melhor entender suas atividades e contribuições, são classificados de acordo com seu tamanho, em microfauna, mesofauna e macrofauna. Dentre as funções essenciais para a manutenção e sobrevivência de comunidades vegetais e animais nos ecossistemas, desempenhada pelos diferentes organismos existentes no solo, a meso e a macrofauna se destacam por exercerem papel relevante na trituração, distribuição do material orgânico e promoção de alterações substanciais nos parâmetros físicos, tais com porosidade e agregação do solo (GARDI et al., 2009).

Os organismos da mesofauna destacam-se por apresentar funcionalidade alimentar diferente, através do consumo de microrganismos e da fragmentação da serapilheira. Contribuem nos processos de recuperação e restauração do solo, uma vez que agem na ciclagem de nutrientes, estímulo e controle dos microrganismos, além de servirem de alimento para outros grupos da fauna (OLIVEIRA FILHO et al., 2015).

Da mesma forma, a macrofauna edáfica tem seu benefício cada vez mais reconhecido pelo papel ativo que desempenha no crescimento das plantas, na manutenção dos teores de matéria orgânica do solo e na melhoria das propriedades físicas, por atuarem na estruturação do solo. Além disso, contribuem com a manutenção da qualidade do solo, pois, por meio da sua movimentação e atividades, realizam a redistribuição da matéria orgânica ao longo do perfil (BARETTA; BROWN; CARDOSO, 2010).

A fragmentação dos habitats e a conversão de áreas naturais em sistemas de produção podem modificar a densidade e diversidade dos grupos mais frequentes de organismos edáficos. Essas mudanças observadas nos ecossistemas ocorrem devido a intensificação de uso da terra, que provoca um impacto desproporcional sobre a biota do solo, pois a redução da biodiversidade pode não ser uniforme em todos os táxons (LIIRI et al., 2012). Estes mesmos autores, salientam que os invertebrados se mostram sensíveis a diferentes níveis de intervenção no ambiente, refletindo o quanto determinada prática de manejo realmente conserva o solo do ponto de vista da estrutura e da fertilidade.
Frente ao exposto, por ser um fator dinâmico e por estar intimamente associada aos processos e perturbações no solo, recentemente a fauna edáfica tem sido vista como bioindicadora de sua qualidade, e desta forma apresenta capacidade de sinalizar antecipadamente informações sobre a situação desse ambiente. As características de resposta da fauna edáfica por si só já servem de base para estudos que avaliam as modificações ocorridas no solo (JÓZEFOWSKA; WOŚ; PIETRZYKOWSKI, 2016). Portanto, o objetivo do presente estudo foi avaliar a diversidade dos grandes grupos taxonômicos de invertebrados da meso e macrofauna do solo e suas relações com atributos físicos e químicos em diferentes usos do solo, envolvendo pastagem nativa melhorada, reflorestamento de Pinus sp. e floresta nativa em Lages, SC.

\section{MATERIAL E MÉTODOS \\ Caracterização da área de estudo}

O presente estudo foi desenvolvido em diferentes usos da terra em áreas pertencentes à Empresa de Pesquisa Agropecuária e Extensão Rural de Santa Catarina (EPAGRI), no município de Lages, Planalto Catarinense. O clima da região é temperado (Köppen, $\mathrm{Cfb}$ ), com chuvas uniformemente distribuídas e precipitação anual média variando de 1100 a $2000 \mathrm{~mm}$, sem estação seca e com temperatura média do mês mais quente não atingindo 22 ${ }^{\circ} \mathrm{C}$, com um verão fresco. Também ocorrem geadas severas e frequentes, ocorrendo no mínimo vinte e cinco vezes por ano (ALVARES et al., 2013).

No local de estudo, foram avaliados diferentes usos da terra (Tabela 1), sendo: 1) Campo nativo melhorado (CNM), implantado em remanescente de campo nativo, no qual havia resíduos vegetais (palha grossa), sendo também, recentemente, introduzidas espécies forrageiras hibernais Lolium multiflorum L. (azevémanual), Avena strigosa Schreb. (aveia-preta), Trifolium repens $\mathrm{L}$. (trevo-branco), Trifolium pratense L. (trevo-vermelho); 2) reflorestamento de pinus (RP), no qual foi implantado Pinus sp. há 23 anos em área de campo nativo, em espaçamento de plantio de $2,5 \times 2,5 \mathrm{~m}$, sem intervenções de desbastes sobre o povoamento e havendo livre acesso de animais (bovinos); 3) floresta nativa (FN), remanescente de Floresta Ombrófila Mista em estágio secundário de sucessão, o qual não foi submetido à intervenção antrópica nos últimos seis anos e também, sem acesso de animais. 
Tabela 1. Características dos sistemas de floresta nativa (FN), reflorestamento de pinus (RP) e campo nativo melhorado (CNM) no município de Lages, SC.

\begin{tabular}{cccc}
\hline Sistemas & Área (ha) & Coordenadas & Altitude (m) \\
\hline \multirow{2}{*}{ FN } & 3 & $\mathrm{~S} 27^{\circ} 48.247^{\prime}$ & 954 \\
& & $\mathrm{~W} 50^{\circ} 20.080^{\prime}$ & \\
\multirow{2}{*}{$\mathrm{RP}$} & 1 & $\mathrm{~S} 27^{\circ} 48.237$ & 950 \\
& & $\mathrm{~W} 50^{\circ} 20.051^{\prime}$ & \\
$\mathrm{CNM}$ & 4,6 & $\mathrm{~S} 27^{\circ} 48.103^{\prime}$ & 975 \\
& & $\mathrm{~W} 50^{\circ} 20.119^{\prime}$ & \\
\hline
\end{tabular}

\section{Amostragem}

Em cada uso da terra foram coletadas amostras para a determinação das propriedades químicas e físicas do solo e diversidade da meso e macrofauna edáfica, no mês de outubro de 2015, delimitando-se em cada uso três transectos distanciados $30 \mathrm{~m}$ entre si.

A macrofauna edáfica foi amostrada pelo método "Tropical Soil Biology and Fertility" (TSBF) (ANDERSON; INGRAM, 1993). Este método consiste na coleta de monólitos de solo de 25 × $25 \mathrm{~cm}$ de largura e $20 \mathrm{~cm}$ de profundidade, com auxílio de um marcador constituído de chapas de ferro galvanizadas. Os monólitos coletados foram ensacados, levados ao laboratório de solos, onde procedeu-se a triagem manual, sendo os organismos visíveis a olho nu separados em frascos plásticos contendo solução de álcool 70\% para preservação. Posteriormente, os organismos foram identificados em nível de grandes grupos taxonômicos (ordem), com auxílio de microscópio estereoscópico.
O outro método utilizado para amostragem da fauna edáfica, foram as armadilhas do tipo "Pitfall traps", que embora sejam adequadas para amostrar organismos da mesofauna capturam também organismos pertencentes à macrofauna. Este método consiste na implantação de recipientes cilíndricos com abertura de $8 \mathrm{~cm}$ de diâmetro, com capacidade volumétrica de $500 \mathrm{~mL}$, contendo $200 \mathrm{~mL}$ de solução detergente a $0,5 \%(\mathrm{v} / \mathrm{v})$ e enterrados com sua extremidade vazada nivelada com a superfície do solo (BARETTA et al., 2014). Esses recipientes foram mantidos a campo por três dias consecutivos (72 h) e, então retirados, fechados e levados ao laboratório, onde foi realizado o peneiramento das amostras em malhas 2 e 0,100 mm. Posteriormente os organismos capturados foram identificados a nível de ordem da mesma forma que no método TSBF.

Para determinação das propriedades químicas do solo foram coletados em cada ponto de avaliação da fauna quatro sub-amostras na camada de 0 a $10 \mathrm{~cm}$ de profundidade com auxílio de trado holandês para as determinações de $\mathrm{pH}$ em água, índice SMP, $\mathrm{P}, \mathrm{K}, \mathrm{Al}^{3+}$, $\mathrm{Ca}^{2+}, \mathrm{Mg}^{2+}, \mathrm{MO}, \mathrm{H}+\mathrm{Al}, \mathrm{CTC}_{\mathrm{pH}} 7.0$ e soma de bases, conforme metodologia de Tedesco et al. (1995) (Tabela 2). Além das propriedades químicas do solo, também foram determinas: a resistência do solo à penetração (RPen) à campo, utilizando um penetrômetro eletrônico e automatizado, umidade do solo (Umi) com um medidor eletrônico de umidade volumétrica do solo e o teor de Clorofila Total (ClorT) das plantas mais comuns, com o clorofilômetro eletrônico em cada uso da terra, conforme a Tabela 2. 
Tabela 2. Valores médios \pm desvio padrão dos atributos químicos e físicos do solo na camada de $0-10 \mathrm{~cm}$, nos sistemas de floresta nativa (FN), reflorestamento de pinus (RP) e campo nativo melhorado (CNM) no município de Lages, SC.

\begin{tabular}{lccc}
\hline \multirow{2}{*}{ Variável } & & Uso da terra & CNM \\
\cline { 2 - 4 } & $53,73 \pm 4,71 \mathrm{a}$ & $\mathrm{RP}$ & $55,9 \pm 2,62 \mathrm{a}$ \\
\hline Umi $(\%)$ & $28,8 \pm 12,91 \mathrm{a}$ & $21,1 \pm 6,11 \mathrm{a}$ & $36,63 \pm 5,42 \mathrm{a}$ \\
ClorT & $1386,59 \pm 669,84 \mathrm{a}$ & $699,67 \pm 426,07 \mathrm{a}$ & $1247,92 \pm 34,32 \mathrm{a}$ \\
RPen $(\mathrm{KPa})$ & $4,2 \pm 0,21 \mathrm{~b}$ & $4,9 \pm 0,24 \mathrm{a}$ & $5,2 \pm 0,26 \mathrm{a}$ \\
$\mathrm{pH}$ & $4,7 \pm 0,23 \mathrm{~b}$ & $5,2 \pm 0,26 \mathrm{~b}$ & $6,1 \pm 0,30 \mathrm{a}$ \\
$\mathrm{pHSMP}$ & $6,9 \pm 0,34 \mathrm{a}$ & $5,1 \pm 0,25 \mathrm{~b}$ & $4,6 \pm 0,23 \mathrm{~b}$ \\
$\mathrm{P}\left(\mathrm{mg} \mathrm{dm}^{-3}\right)$ & $72 \pm 3,6 \mathrm{c}$ & $108 \pm 5,4 \mathrm{~b}$ & $168 \pm \mathrm{a}$ \\
$\mathrm{K}\left(\mathrm{mg} \mathrm{dm}^{-3}\right)$ & $3 \pm 0,15 \mathrm{~b}$ & $2,8 \pm 0,14 \mathrm{~b}$ & $3,8 \pm 0,19 \mathrm{a}$ \\
$\mathrm{MO}(\%)$ & $5,6 \pm 0,28 \mathrm{a}$ & $5,3 \pm 0,26 \mathrm{a}$ & $1,7 \pm 0,08 \mathrm{~b}$ \\
$\mathrm{Al}\left(\mathrm{cmol}_{\mathrm{c}} \mathrm{dm}^{-3}\right)$ & $0,1 \pm 0,01 \mathrm{c}$ & $0,4 \pm 0,02 \mathrm{~b}$ & $2,2 \pm 0,11 \mathrm{a}$ \\
$\mathrm{Ca}\left(\mathrm{cmol}_{\mathrm{c}} \mathrm{dm}^{-3}\right)$ & $0,2 \pm 0,01 \mathrm{c}$ & $0,7 \pm 0,03 \mathrm{~b}$ & $1,6 \pm 0,08 \mathrm{a}$ \\
$\mathrm{Mg}\left(\mathrm{cmol}_{\mathrm{c}} \mathrm{dm}^{-3}\right)$ & $19,63 \pm 0,98 \mathrm{a}$ & $11,05 \pm 0,55 \mathrm{~b}$ & $4,02 \pm 0,20 \mathrm{c}$ \\
$\mathrm{HAl}\left(\mathrm{cmol}_{\mathrm{c}} \mathrm{dm}^{-3}\right)$ & $20,09 \pm 1,00 \mathrm{a}$ & $12,44 \pm 0,62 \mathrm{~b}$ & $8,27 \pm 0,41 \mathrm{c}$ \\
$\mathrm{CTC}$ & $2,28 \pm 0,11 \mathrm{c}$ & $11,19 \pm 0,56 \mathrm{~b}$ & $51,41 \pm 2,57 \mathrm{a}$ \\
SB $\left(\mathrm{CTC}^{1}\right)$ & &
\end{tabular}

Umi: umidade; ClorT: clorofila total das plantas rasteiras; RPen: resistência a penetração; pH: potencial hidrogeniônico; P: fósforo; K: potássio; MO: matéria orgânica; Al: alumínio; Ca: cálcio; Mg: magnésio; HAl: acidez potencial; CTC: capacidade de trocar cátions a pH 7,0; SB: soma de bases trocáveis; 1\% de saturação na CTC a pH 7,0. Médias seguidas da mesma letra nas linhas não diferem significativamente pelo teste de Tukey ( $\mathrm{p} \leq 0,05)$.

\section{Análise dos dados}

Os dados de abundância da fauna edáfica foram submetidos à análise multivariada, sendo realizada uma Detrended Correspondence Analysis (DCA), a fim de obter o comprimento do gradiente. Tendo em vista que o comprimento do gradiente foi inferior a três (resposta linear), realizou-se Análise de Componentes Principais (ACP).

Para verificar a hipótese de relação entre as variáveis ambientais e a fauna do solo, os dados foram submetidos a Análise de Redundância (RDA). Nesta, as variáveis explicativas colineares foram identificadas através do Variance Inflation Factor (VIF), e por operações de forward selection, usando sucessivas RDAs com base em permutações por teste de Monte-Carlo, sendo retiradas as que apresentaram colinearidade e selecionadas as que melhor explicaram a variação dos dados $(p \leq 0,05)$. Esse método permitiu a escolha de um conjunto mínimo de variáveis físicas e químicas significativas para explicar a variação da fauna em cada uso da terra. As variáveis significativas das RDAs foram posteriormente utilizadas nas ACPs como variáveis ambientais explicativas passivas para as mudanças observadas nos grupos da fauna. Para as análises utilizou-se o software estatístico CANOCO versão 4.5 (ter BRAAK; SMILAUER, 2002).

Os índices de equabilidade de Pielou (J) e diversidade de Shannon-Wiener $\left(\mathrm{H}^{\prime}\right)$ foram calculados através da biblioteca VEGAN ( $\mathrm{R}$ DEVELOPMENT CORE TEAM, 2011) presentes no programa estatístico $R$. Os atributos ambientais foram submetidos a análise de variância e o teste Post-Hoc de Tukey para comparações múltiplas nos três diferentes usos da terra, utilizando o programa estatístico SPSS v. 20 (SPSS IBM, 2011).

\section{RESULTADOS E DISCUSSÃO}

Riqueza e diversidade da fauna edáfica

Foram identificados 344 indivíduos pelo método TSBF e 866 por Pitfall traps, totalizando 1.210 indivíduos, distribuídos em 17 grupos taxonômicos (Tabela 3), sendo os grupos mais representativos Collembola (36\%), 
Tabela 3. Abundância dos grupos taxonômicos da fauna edáfica amostrada por TSBF e Pitfall traps, índices de diversidade de Shannon-Wiener (H') e equabilidade de Pielou (J) e riqueza, nos sistemas de floresta nativa (FN), reflorestamento de pinus (RP) e campo nativo melhorado (CNM) no município de Lages, SC.

\begin{tabular}{|c|c|c|c|c|c|c|c|c|c|}
\hline \multirow[b]{2}{*}{ Grupos } & \multicolumn{3}{|c|}{ TSBF } & \multicolumn{3}{|c|}{ Pitfall traps } & \multicolumn{3}{|c|}{ TSBF+Pitfall } \\
\hline & $\mathrm{FN}$ & $\mathrm{RP}$ & $\mathrm{CNM}$ & $\mathrm{FN}$ & $\mathrm{RP}$ & $\mathrm{CNM}$ & $\mathrm{FN}$ & $\mathrm{RP}$ & $\mathrm{CNM}$ \\
\hline Collembola & 0 & 0 & 0 & 123 & 104 & 204 & 123 & 104 & 204 \\
\hline Coleoptera & 37 & 9 & 70 & 13 & 3 & 89 & 50 & 12 & 159 \\
\hline Hymenoptera & 74 & 8 & 13 & 39 & 4 & 70 & 113 & 12 & 83 \\
\hline Acarina & 0 & 0 & 0 & 26 & 17 & 53 & 26 & 17 & 53 \\
\hline Diptera & 0 & 0 & 0 & 39 & 11 & 17 & 39 & 11 & 17 \\
\hline Enchytraeidae & 2 & 26 & 23 & 0 & 0 & 0 & 2 & 26 & 23 \\
\hline Araneae & 6 & 1 & 6 & 2 & 1 & 23 & 8 & 2 & 29 \\
\hline Hemiptera & 6 & 0 & 0 & 3 & 1 & 10 & 9 & 1 & 10 \\
\hline Oligochaeta & 0 & 3 & 15 & 0 & 0 & 0 & 0 & 3 & 15 \\
\hline Outros $^{1}$ & 6 & 6 & 4 & 2 & 0 & 0 & 8 & 6 & 4 \\
\hline Blattodea & 4 & 4 & 0 & 3 & 2 & 0 & 7 & 6 & 0 \\
\hline Isoptera & 7 & 0 & 0 & 1 & 2 & 0 & 8 & 2 & 0 \\
\hline Diplopoda & 4 & 4 & 0 & 0 & 0 & 0 & 4 & 4 & 0 \\
\hline Diplura & 0 & 0 & 0 & 3 & 0 & 0 & 3 & 0 & 0 \\
\hline Chilopoda & 3 & 0 & 0 & 0 & 0 & 0 & 3 & 0 & 0 \\
\hline Opiliones & 0 & 1 & 0 & 1 & 0 & 0 & 1 & 1 & 0 \\
\hline Isopoda & 1 & 0 & 0 & 0 & 0 & 0 & 1 & 0 & 0 \\
\hline Gastropoda & 0 & 1 & 0 & 0 & 0 & 0 & 0 & 1 & 0 \\
\hline Abundância & 150 & 63 & 131 & 255 & 145 & 466 & 405 & 208 & 597 \\
\hline Riqueza & 11 & 10 & 6 & 12 & 9 & 7 & 16 & 15 & 10 \\
\hline $\mathrm{H}^{\prime}$ & 1,58 & 1,36 & 1,36 & 1,59 & 1,05 & 1,56 & 1,94 & 1,80 & 1,78 \\
\hline $\mathrm{J}$ & 0,6 & 0,52 & 0,52 & 0,62 & 0,41 & 0,61 & 0,66 & 0,61 & 0,60 \\
\hline
\end{tabular}

Coleoptera $(18 \%)$ e Hymenoptera (17\%), independente do método e uso da terra. A abundância dos indivíduos da fauna amostrada por TSBF, quando convertida para densidade por metro quadrado (ind. $\mathrm{m}-2$ ), apresentou densidade média de 612 ind. m-2. A densidade máxima foi de 1.584 ind. $\mathrm{m}^{-2}$ em FN e a mínima 208 ind. $\mathrm{m}^{-2} \mathrm{em} \mathrm{RP}$.

$\mathrm{O}$ maior valor de diversidade de grupos $\mathrm{H}^{\prime}$ foi observado em FN (Tabela 3), nos dois métodos de amostragem quando avaliados separadamente ou juntos. $\mathrm{O}$ mesmo padrão foi observado para os valores do índice $\mathrm{J}$. Rosa et al. (2015) encontraram valores semelhantes para H', na região do Planalto Sul Catarinense, com amostragem da fauna pelo método TSBF, onde o maior valor coincidiu com o sistema FN. Bartz et al. (2014) também observaram esse padrão ao estudar a abundância da fauna edáfica pelo método Pitfall traps na região Oeste de Santa Catarina, o que reforça os dados apresentados no presente estudo.

\section{Análise de Composição da comunidade}

A ACP (Figura 1) considerando as abundâncias totais dos grupos, independente de método de amostragem demonstrou distinção entre os usos da terra, através da relação entre a componente principal 1 (CP1) e a componente principal 2 (CP2). A CP1 explicou 33,0\% e a CP2 explicou $25,2 \%$, totalizando $58,2 \%$ da variabilidade dos dados. Nesta, a maior parte dos grupos da fauna ficaram mais associados ao sistema $\mathrm{FN}$, seguida do $\mathrm{CNM}$, enquanto o RP ficou mais isolado e com baixa quantidade de grupos associados.

Os grupos Diptera, Chilopoda e Isoptera foram mais abundantes no sistema $\mathrm{FN}$ em comparação aos demais uso da terra (Figura 1). Segundo Marichal et al. (2014), esses grupos são geralmente associados a abundância e qualidade de serapilheira. 


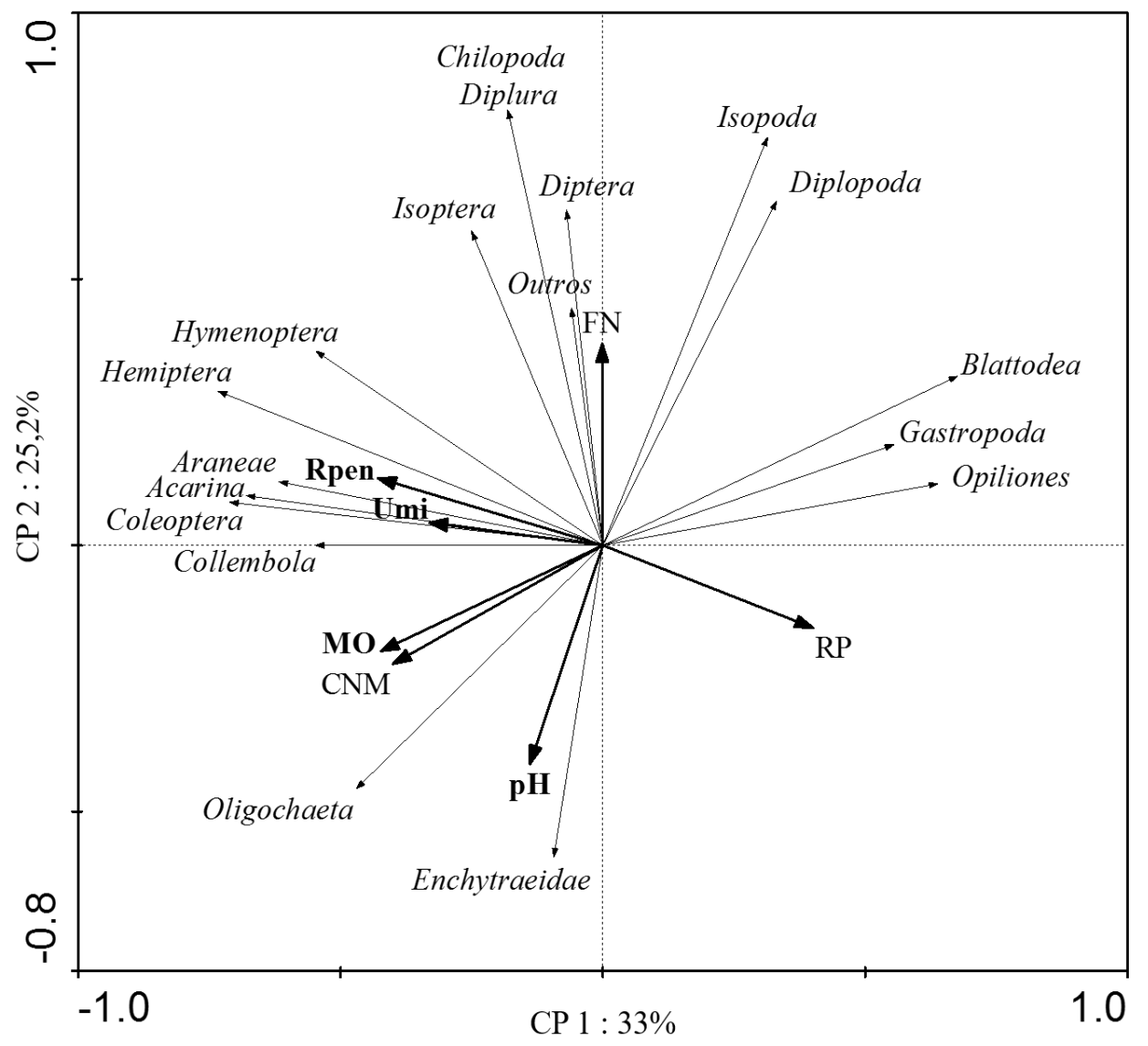

Figura 1. Análise de componentes principais (ACP) para os grupos da fauna edáfica (itálico), independente de método de amostragem (Soma de TSBF e armadilhas) e as variáveis ambientais (negrito) utilizadas como explicativas, nos sistemas de floresta nativa (FN), reflorestamento de pinus (RP) e campo nativo melhorado (CNM) no município de Lages, SC. Abreviações: Umidade (Umi); Matéria Orgânica (MO); Resistência a penetração (Rpen); potencial hidrogeniônico (pH).

Os grupos Oligochaeta, Colembolla e Enchytraeidae foram mais abundantes no sistema CNM, enquanto Opiliones e Gastropoda no sistema RP.

O microclima e a intensidade do uso do solo afetam os organismos edáficos e por isso o maior número de grupos encontrados em FN pode estar relacionado com esses fatores. Além disso na FN a cobertura vegetal mantém-se inalterada, o que consequentemente garante menores variações de temperatura, proporcionando ambiente mais favorável para o estabelecimento de vários grupos de organismos edáficos. Outro aspecto importante da FN é sua maior diversidade florística, que promove a formação de uma serapilheira mais diversa, ou seja, oferece variedade de alimento e substrato para a fauna do solo. Diferente do que verifica-se em monoculturas florestais, como é o caso em RP, que possuem serapilheira proveniente de uma única espécie e, portanto, de baixa qualidade nutricional para os organismos do solo (MAESTRI et al., 2013).

Contudo, a diversidade da fauna edáfica depende de muitos fatores, como tipo de manejo, disponibilidade de fontes de alimento, teor de matéria orgânica e umidade do solo e, desta forma, ficam suscetíveis às mudanças microclimáticas do hábitat.

De acordo com os resultados apresentados na RDA verificou-se que existe interação entre a fauna edáfica e os atributos físicos e químicos avaliados. O estudo de Baretta et al. (2014) também demonstrou a ligação entre os invertebrados e as propriedades do solo e o quanto esses fatores são afetados pelo manejo. 
A RDA (Figura 2) indicou que as variáveis ambientais explicam $79,1 \%$ da variabilidade dos dados, sendo desta porção, 47,2\% explicado pela CP 1 e 31,9\% pelo eixo da CP 2 (Figura 2). Quando observadas as relações dos atributos físicos e químicos com a fauna, os maiores teores de $\mathrm{MO}$ e $\mathrm{pH}$ se correlacionaram com os grupos Collembola, Oligochaeta e Enchytraeidae, sendo fatores determinantes para o desenvolvimento desses

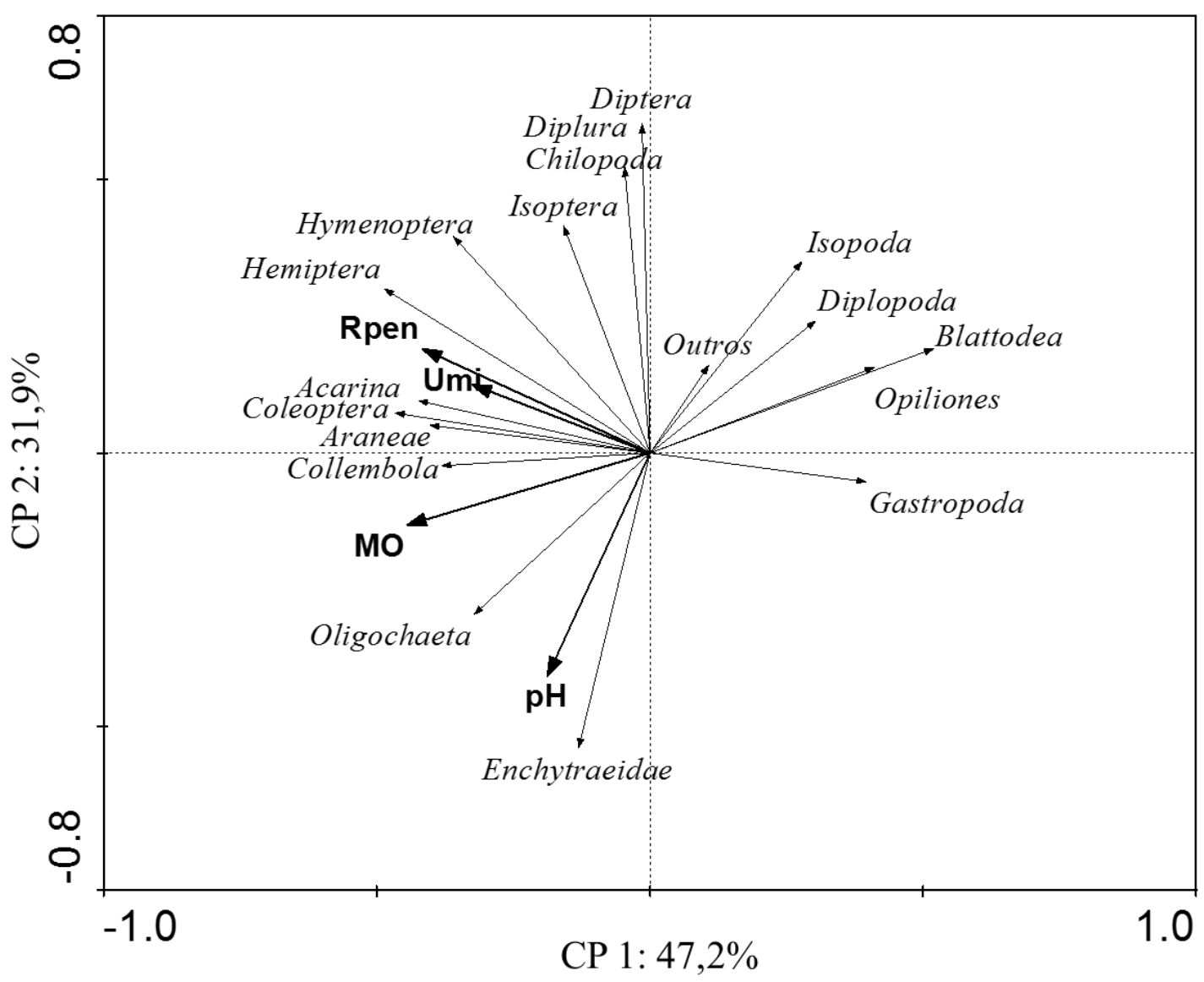

Figura 2. Análise de redundância (RDA) para os grupos da fauna edáfica (itálico) e os atributos físicos e químicos do solo (negrito), independente de método de amostragem (Soma de TSBF e armadilhas) e dos usos da terra, no município de Lages, SC. Abreviações: Umidade (Umi); Matéria Orgânica (MO); Resistência a penetração (Rpen); potencial hidrogeniônico (pH).

grupos (disponibilidade de recursos alimentares). No caso do CNM ressalta-se que os maiores teores de MO podem estar relacionados a deposição de sobras energéticas (esterco) do gado presente neste sistema, mas principalmente pelos resíduos gerados da cobertura vegetal e raízes (Figura 1 e Tabela 2). Os atributos Umi e Rpen foram associados também na ACP aos dois sistemas FN e CNM (Figura 1 e Tabela 2), assim como a maior parte dos grupos da fauna, especialmente os grupos Coleoptera, Acarina, Araneae, Hemiptera e Hymenoptera.
A umidade é um dos parâmetros do solo que garante a disponibilidade de recursos para grupos como ácaros e enquitreídeos (Enchytraeidae), no entanto, pelo fato de apresentarem pouca mobilidade no solo, os índices de umidade podem ser fatores limitantes para o estabelecimento destes no solo, pois em condições extremas o forrageamento será limitado. Harada e Bandera (1994), estudando três coberturas vegetais na Amazônia Central, verificaram que a maioria dos grupos de fauna do solo apresentaram uma correlação positiva com a umidade do solo, ratificando a ideia de que uma cobertura vegetal 
de qualidade e quantidade, garante disponibilidade de recursos adequados para o estabelecimento da fauna do solo.

A temperatura é o principal fator que influencia na regulação metabólica nos indivíduos edáficos, e juntamente com a umidade, determinam sua distribuição espacial e os períodos de atividade elevada. Os colêmbolos (Collembola), por exemplo, são invertebrados nos quais essas condições microclimáticas, determinam o habitat ideal e controlam a taxa de reprodução e crescimento dos indivíduos e sua distribuição vertical ao longo de um perfil. Esse grupo também atua na decomposição da matéria orgânica e o acúmulo de resíduos orgânicos nesses usos, fatores que influenciam diretamente na entrada e manutenção da água no solo, tornando o meio adequado para a sobrevivência de outros organismos edáficos (VERMA; YADAV; KUMAR, 2014).

Além disso, alterações no $\mathrm{pH}$ interferem na diversidade dos colêmbolos, pois a presença ou ausência de algumas espécies pode estar relacionada com a disponibilidade de íons específicos na água. Portanto, o manejo realizado em áreas agrícolas, com a finalidade de melhorar a fertilidade e diminuir a acidez do solo, provoca variações no $\mathrm{pH}$ que podem causar estresse nessas comunidades (SILVA et al., 2015).

No presente estudo o grupo Coleoptera apresentou relação com o atributo Umidade (Figura 2), resultado este também observado por Vasconcellos et al. (2013). Algumas famílias dessa ordem sofrem alterações na sua comunidade principalmente decorrentes de mudanças microclimáticas, sendo sensíveis indicadores de temperatura e umidade (GARLET et al., 2015). Além disso, os decompositores primários (Molusca, Blattodea e Isopoda) estão mais relacionados a FN (Figuras 1 e Tabela 3) em função da diversidade e quantidade de material vegetal depositado.

As minhocas (Oligochaeta), estão envolvidas na decomposição de matéria orgânica e ciclagem de nutrientes e têm potencial de influenciar substancialmente alguns processos dos ecossistemas, colaborando para o favorecimento do habitat e enriquecimento da diversidade edáfica (ANDRÉA, 2010).

Os enquitreídeos, possuem ampla distribuição tanto em solos naturais florestais e pastagens quanto em solos cultivados ou com outro tipo de intervenção antrópica. São pouco tolerantes a falta de umidade e a variações de temperatura, sendo, fatores importantes na distribuição do grupo. Além destes, a matéria orgânica, $\mathrm{pH}$ e o uso do solo podem influenciar a composição de espécies de enquitreídeos (NIVA et al., 2010).

No CNM a maior quantidade de MO encontrada, deve-se a abundância de resíduos vegetais encontrados na superfície do solo. Outro aspecto importante refere-se as práticas de manejo a calagem, comumente empregada no melhoramento do campo nativo, que aumenta o valor do atributo químico $\mathrm{pH}$. Os maiores valores desses atributos, indicados na Tabela 2, contribuem para explicar a maior afinidade dos referidos grupos com CNM na ACP (Figura 1).

A RDA (Figura 2) mostra a proximidade entre a Rpen e Umidade. Sabe-se, que a umidade altera a coesão entre as partículas do solo. Desta forma, quando o solo se encontra seco ou com baixo conteúdo de água, suas partículas apresentam-se mais próximas e difíceis de serem separadas por forças externas, à medida que o solo começa a umedecer a partículas sedem mais facilmente a aplicação de força. Frente a isso, o fato dessas variáveis aumentarem seguindo a mesma orientação na ordenação, contrapõe a afirmação supracitada e indica que o aumento da Rpen possivelmente está relacionado a outro fator ambiental presente no sistema, como a maior densidade do solo que pode ser influenciada por pisoteio provocado pela presença de bovinos no CNM.

\section{CONCLUSÃO}

A fauna edáfica é influenciada pelos atributos químicos e físicos do solo, fatores estes que explicam a diversidade e abundância dos grupos distribuídos entre a Floresta Nativa (FN), Campo Nativo Melhorado (CNM) e Reflorestamento de Pinus (RP) no município de Lages, SC.

Dentre os usos da terra, o RP reduz a abundância dos grupos da fauna, enquanto $\mathrm{FN}$ e CNM apresentam maior biodiversidade edáfica. Maiores teores de matéria orgânica e $\mathrm{pH}$ apresentaram relação com Oligochaeta, Enchytraeidae e Collembola, independente do uso da terra avaliado.

Quando o objetivo for avaliar a diversidade de grupos da fauna edáfica, a utilização conjunta dos dois métodos, de monólitos (TSBF) e armadilhas (Piffall traps), pode minimizar as limitações amostrais decorrente de cada método. Sendo assim, a realização de futuros estudos que objetivam a avaliação da fauna edáfica, com uso de diferentes metodologias amostrais e analisem a real diferença entre a utilização destas separadas ou em conjunto, podem contribuir para elucidar a melhor forma de análise dos invertebrados do solo.

\section{AGRADECIMENTOS}

Aos programas de Pós-graduação em Ciência do Solo e em Engenharia Florestal da UDESC/CAV, Lages, SC. D. Baretta agradece ao CNPq (Conselho Nacional de 
Desenvolvimento Científico e Tecnológico) pela Bolsa de Produtividade Científica do $\mathrm{CNPq}$ (Processo $\mathrm{n}^{\circ}$ 307162/2015-0). À CAPES (Coordenação de Aperfeiçoamento de Pessoal de Nível Superior) e ao PROMOP (Programa de Monitoria de Pós-graduação) pela concessão de bolsas.

\section{REFERÊNCIAS}

ALVARES, C. A.; STAPE, J. L.; SENTELHAS, P. C.; GONÇALVES, J. L. de M.; SPAROVEK, G. Köppen's climate classification map for Brazil. Meteorologische Zeitschrift. v. 22, n. 6, p. 711-728, 2013.

ANDERSON, J. M.; INGRAM, J. S. I. Tropical soil biology and fertility: a handbook of methods. $2^{\text {a }}$. ed. Wallingford: CAB International; 1993.

ANDREA, M. M. O uso de minhocas como bioindicadores de contaminação de solos. Acta Zoológica Mexicana. v. 2, p. 95-107, 2010.

BARDGETT, R. D.; VAN DER PUTTEN, W. H. Belowground biodiversity and ecosystem functioning. Nature. v. 515, n. 7528, p. 505-511, 2014.

BARETTA, D.; BARTZ, M. L. C.; FACHINI, I.; ANSELMI, R.; ZORTÉA, T.; BARETTA, C. D. R. M. Soil fauna and its relation with environmental variables in soil management systems. Revista Ciência Agronômica. v. 45, n. 5 , p. $871-879,2014$.

BARETTA, D.; BROWN, G. G.; CARDOSO, E. J. B. N. Potencial da macrofauna e outras variáveis edáficas como indicadores de qualidade do solo em áreas com Araucaria angustifolia. Acta Zoológica Mexicana. v. 2 p. 135-150, 2010.

BARTZ, M. L. C.; BROWN, G. G.; ORSO, R.; MAFRA, À. L.; BARETTA, D. The influence of land use systems on soil and surface litter fauna in the western region of Santa Catarina. Revista Ciência Agronômica. v. 45, n. 5, p. 880-887, 2014.

GARDI, C.; MONTANARELLA, L.; ARROUAYS, D.; BISPO, A.; LEMANCEAU, P.; JOLIVET, C.; MULDER, C.; RANJARD, L.; RÖMBKE, J.; RUTGERS, M.; MENTA, C. Soil biodiversity monitoring in Europe:

OLIVEIRA FILHO, L. C. I.; BARETTA, D.; VIAPIANA, C. M.; SANTOS, J. C. P. Mesofauna de solo construído em área de mineração de carvão. Revista de Ciências Agroveterinárias. v. 14, n. 1, p. 55-64, 2015. ongoing activities and challenges. European Journal of Soil Science. v. 60, n. 5, p. 807-819, 2009.

GARLET, J.; COSTA, E. C.; BOSCARDIN, J.; MACHADO, D. N.; PEDRON, L. Fauna de coleóptera edáfica em eucalipto sob diferentes sistemas de controle químico da mato competição. Floresta e Ambiente. v. 22, n. 2, p. 239-248, 2015.

HARADA, A. Y.; BANDEIRA, J. M. Estratificação e densidade de invertebrados em solo arenoso sob floresta primária e plantios arbóreos na Amazônia Central durante a estação seca. Acta amazônica. v. 24, n. 1-2, p. 103-118, 1994.

JÓZEFOWSKA, A.; WOŚ, B.; PIETRZYKOWSKI, M. Tree species and soil substrate effects on soil biota during early soil forming stages at afforested mine sites. Applied Soil Ecology. v. 102, p. 70-79, 2016.

LIIRI, M.; HÄSÄ, M.; HAIMI, J.; SETÄLÄ, H. History of landuse intensity can modify the relationship between functional complexity of the soil fauna and soil ecosystem services - A microcosm study. Applied Soil Ecology. v. 55, p. 53-61, 2012.

MAESTRI, R.; LEITE, M.; SCHMITT, L.; RESTELLO, R. Efeito de mata nativa e bosque de eucalipto sobre a riqueza de artrópodos na serapilheira. Perspectiva. v. 37, p. 31-40, 2013.

MARICHAL, R.; GRIMALDI, M.; FEIJOO, A. M.; OSZWALD, J.; PRAXEDES, C.; RUIZ COBO, D. H.; HURTADO, M. Del P.; DESJARDINS, T.; DA SILVA JUNIOR, M. L.; DA SILVA COSTA, L. G.; MIRANDA, I. S.; OLIVEIRA, M. N. D.; BROWN, G. G.; TSÉLOUIKO, S.; MARTINS, M. B.; DECAËNS, T.; VELASQUEZ, E.; LAVELLE, P. Soil macroinvertebrate communities and ecosystem services in deforested landscapes of Amazonia. Applied Soil Ecology. v. 83, p. 177$185,2014$.

NIVA, C. C.; RÖMBKE, J.; SCHMELZ, R. M.; BROWN, G. G. Enquitreídeos (Enchytraeidae, Oligochaeta, Annelida). In: MOREIRA, F. M. S.; HUISING, J.; BIGNELL, D. E. 2a . ed. Manual de Biologia dos Solos Tropicais: Amostragem e caracterização da biodiversidade. Lavras: UFLA. p.351-361, 2010.

R DEVELOPMENT Core Team. R: a language and environment for statistical computing. [2.12.2]. Vienna: R Foundation for Statistical Computing, 2011. 
ROSA, M. G.; KLAUBERG FILHO, O.; BARTZ, M. L. C.; MAFRA, Á. L.; SOUSA, J. P. F. A. de; BARETTA, D. Macrofauna edáfica e atributos físicos e químicos em sistemas de uso do solo no Planalto Catarinense. Revista Brasileira de Ciência do Solo. v. 39, p. 1544-1553, 2015.

SILVA， P. M.; CARVALHO, F.; DIRILGEN, T.; STONE, D.; CREAMER, R.; BOLGER, T.; SOUZA, J. P. Traits of collembolan life-form indicate land use types and soil properties across an European transect. Applied Soil Ecology. v. 97, p. 69-77, 2015.

SPSS IBM. IBM SPSS statistics base 20. Chicago, IL: SPSS Inc. 2011.

TEDESCO, M. J.; GIANELLO, C.; BISSANI, C. A.; BOHNEN, H.; VOLKWEISS, S. J. Análise de solo, plantas e outros materiais. $2^{\text {a }}$. ed. Porto Alegre: Universidade Federal do Rio Grande do Sul; 1995.

ter BRAAK, C. J. F.; SMILAUER, P. CANOCO reference manual and CanoDraw for Windows user's guide: software for canonical community ordination (version 4.5). Ithaca: Microcomputer Power; 2002.

VASCONCELLOS, R. L. F.; SEGAT, J. C.; BON, J. A.; BARETTA, D.; CARDOSO, E. J. B. N. Soil macrofauna as an indicator of soil quality in an undisturbed riparian forest and recovering sites of different ages. European Journal of Soil Biology. v. 58, p. 105-112, 2013.

VERMA, D.; YADAV, R. K.; KUMAR, M. Effect of ecological factors on population density of collembolan. Agra. Journal of Environmental and Applied Bioresearch. v. 2, p. 25-28, 2014. 